\title{
Mouzelis y Alexander: lecturas críticas del esquema evolucionista de Talcott Parsons
}

\author{
Natalio Pagés* y Nicolás Rubí**
}

\author{
Categoría: Monografía \\ Fecha de recepción: 28 de mayo de 2012 \\ Fecha de aprobación: 12 de julio de 2012
}

\begin{abstract}
Resumen
En la última etapa de su producción teórica, Parsons concentra sus esfuerzos en la construcción de un sistema general que permita pensar la evolución de las sociedades humanas. Propone el concepto de comunidad societal para resolver el problema de la integración del sistema social ante un contexto moderno de procesos de cambio que promueven creciente diferenciación institucional y pluralidad cultural (potenciación del conflicto). En este artículo nos encargaremos de rastrear las lecturas críticas de Alexander y Mouzelis sobre el esquema parsoniano del cambio social, para identificar los núcleos problemáticos que ambos observan y evaluar hasta qué punto sus propuestas logran escapar de los conflictos del marco evolucionista y despejar a la comunidad societal de sus tensiones internas.

Palabras clave: Comunidad Societal - Evolucionismo - Diferenciación Cambio social
\end{abstract}

\begin{abstract}
In the last stage of his theoretical production, Parsons develops a general system that tries to explain the evolution of human societies. He proposes the notion of Societal Community to solve the problem of Integration within a Social System that presents developed institutional differentiation and cultural plurality. In this article, we will trace the critical readings of Alexander and Mouzelis on the parsonian scheme of Social Change. We'll approach the theoretical problems that both of them observe and evaluate their proposal. Our main task is to find out if Alexander and Mouzelis manage to avoid the difficulties of the evolutionist framework and liberate the Societal Community from its inner tensions.
\end{abstract}

Keywords: Societal Community - Evolutionism - Differentiation - Social Change

* Lic. en Sociología, Facultad de Ciencias Sociales (UBA). Asistente de Coordinación del relevamiento "Impacto de la Asignación Universal por Hijo" (Ministerio de Desarrollo Social), Observatorio de Condiciones de Vida (UNDAV). Estudiante de Realización Cinematográfica (ENERC-INCAA). E-mail: therivertoday@hotmail.com

"* Lic. en Sociología, Facultad de Sociales (UBA). Asistente de Coordinación del relevamiento "Impacto de la Asignación Universal por Hijo" (Ministerio de Desarrollo Social), Observatorio de Condiciones de Vida (UNDAV). Docente de la materia Trabajo Social Comunitario (UNDAV). E-mail: nrubi85@hotmail.com 


\section{Metodología}

Este trabajo surge como resultado de la participación en el grupo de investigación "Teorías sociológicas sobre la comunidad", dirigido por el Dr. Ernesto Pablo de Marinis en el Instituto de Investigaciones Gino Germani (IIGG), Facultad de Ciencias Sociales (UBA), y especialmente del trabajo grupal realizado como parte del proyecto UBACyT "Comunidad y lazo social en la teoría social clásica y contemporánea: un recorrido selectivo por sus derivas y significados". Durante 2010 y comienzo de 2011, se avanzó en un análisis profundo de la obra de Talcott Parsons y el lugar que en ella ocupa la preocupación comunitaria, a partir de su desarrollo de la comunidad societal.

Para ello se realizó un extensivo recorrido por la última etapa de la obra parsoniana y los escritos de numerosos comentaristas contemporáneos (Mouzelis, 1997, 1999; Treviño, 2001; Gerdhardt, 2001, 2002; Chernillo, 2004; Alexander, 2005; Sciortino, 2004, 2005, 2010). En nuestro caso, nos interesamos particularmente por la relación entre la noción de comunidad societal y el desarrollo parsoniano de una teoría evolucionista del cambio social. Sorprendidos por la ausencia de análisis de esa relación en los textos que recuperaban a la comunidad societal para el análisis sociológico contemporáneo, decidimos revisar los estudios críticos sobre el marco evolucionista parsoniano (Lockwood, 1964; Gouldner, 1973; Granovetter, 1979; Fanelli, 1986; Colomy, 1990). Eso nos permitió sintetizar los problemas no resueltos del funcionalismo normativo, como lo llama Lockwood (1964), a la hora de explicar el cambio social. En segunda instancia, regresamos sobre la comunidad societal para observar las dificultades que la noción adquiere al funcionar enteramente dentro de un marco evolucionista.

Luego de una primera presentación de este trabajo en proceso (Pagés y Rubí, 2010), decidimos avanzar en una lectura más profunda de los comentaristas contemporáneos de la obra de Parsons, abocándonos a las pocas perspectivas críticas de la última etapa de su trabajo. Queríamos observar, particularmente, hasta qué pun- 
to había un reconocimiento común de las dificultades de la teoría parsoniana del cambio social, el rol que cumple la comunidad societal en ese marco teórico y las diversas propuestas superadoras al respecto. Encontramos en el trabajo de Nicos Mouzelis (1997, 1999) y Jeffrey Alexander $(1990,2005)$ dos complejas miradas sobre el evolucionismo parsoniano que, lejos de condenarlo superficialmente, profundizan en sus estudios, recuperan muchos de sus conceptos revisándolos críticamente $\mathrm{y}$, a veces, refundan incluso su significado original. Al mismo tiempo, ambos autores comparten la preocupación por el uso descuidado de ciertas nociones para el análisis sociológico de las problemáticas contemporáneas.

Proponemos en este artículo una lectura analítica de ambos autores como una forma de profundizar en nuestro trabajo sobre el marco evolucionista de Talcott Parsons y las tensiones internas de la noción de comunidad societal. En particular, la crítica histórica que propone Mouzelis y la revisión del concepto de "núcleo" que realiza Alexander, nos han permitido comprender con mayor profundidad las razones por las cuales el evolucionismo atenta contra las potencialidades teóricas de la comunidad societal. Este proceso de trabajo, apenas sugerido aquí, nos permitió re-elaborar y profundizar en nuestra primera perspectiva sobre el tema (Pagés y Rubí, 2010). El desarrollo de esta tarea se encuentra plasmado en un nuevo artículo (Pagés y Rubí, 2012) dentro de la compilación colectiva Comunidad: estudios de teoría sociológica (De Marinis, 2012).

\section{Introducción}

El lento proceso de elaboración del concepto de comunidad societal forma parte de un amplio movimiento de renovación teórica que Parsons inicia en mitad de los años ‘50 y termina de sistematizar hacia fines de la década de $1960^{1}$. Para la mayor parte

1 Desarrollos sobre la formación y características de este concepto pueden hallarse en Gerhardt (2001, 2002), Sciortino (2004, 2005, 2010), Alexander (2005) y de Marinis (2010, 2011). 
de sus lectores, el esquema tri-sistémico expuesto en The Social System (1951) proporcionaba un nutrido arsenal de conceptos para analizar, en detalle, la complejidad de cada uno de los sistemas (cultural; social; de la personalidad) bajo un marco abocado a describir niveles, posiciones y funciones de los componentes y unidades, pero era demasiado estático para pensar los intercambios sistémicos y el cambio social. En respuesta a las sucesivas críticas, Parsons comienza a incorporar nuevos elementos que le permitan desarrollar el costado dinámico de su marco analítico.

Por una parte, encuentra en la aplicación de la cibernética al esquema de las cuatro funciones (AGIL) la lógica relacional que necesita para plantear una dinámica de interrelaciones e interpenetraciones entre los subsistemas. De forma simultánea, se interesa crecientemente en la perspectiva evolucionista -decididamente desde Economy and Societies en 1956-; una mirada que se ocupa de sistematizar en diversos trabajos: Evolutionary Universals in Society (1964), Societies (1966) y The System of Modern Societies (1971). Parsons elabora entonces un modelo teórico del cambio social donde los intercambios estructurales son explicados a partir del esquema de los cuatro imperativos funcionales y la historia de las sociedades humanas es organizada en base al desarrollo progresivo de principios universales.

Desde su nuevo marco "cibernético-evolucionista", Parsons propone el concepto de comunidad societal como una forma de resolver el problema de la integración del Sistema Social ante un contexto moderno que promueve creciente diferenciación institucional y pluralidad cultural, es decir, una inminente potenciación de conflictos y tensiones ${ }^{2}$. Sin embargo, los alcances teóricos (y, como veremos, también políticos) de esta noción se ven opacados por una creciente tensión interna entre aspectos particularistas y universalistas. A diferencia del desarrollo parsoniano sobre la So-

2 Para un recorrido por los trabajos parsonianos más importantes en que desarrolla la noción de comunidad societal, véase Parsons (1959, 1965, 1966, 1968, 1971, 2007). 
ciedad (Chernillo, 2004, 2010), donde se diferencia cada nivel de análisis a través de tres nociones (Sistema Social; Sociedad Moderna; Estado-Nación), los distintos usos de la noción de comunidad societal no se encuentran delimitados, definidos, especificados o diferenciados. Esa falta de caracterización de sus múltiples usos y potencialidades puede acarrear importantes complicaciones. Entre ellas, la posibilidad de movilizar argumentos conservadores, como se propone demostrar Alexander (2005).

Hemos intentado ya un primer acercamiento a estas problemáticas (Pagés y Rubí, 2010). Expusimos allí algunos de los problemas generales de la teoría parsoniana del cambio social, recuperando lecturas críticas de su obra y aproximándonos específicamente a sus escritos histórico-políticos. En términos generales, el problema que nos ha convocado es la relación entre el marco teórico del último Parsons y su desarrollo de la comunidad societal. Nos hemos referido, particularmente, a dos tensiones que recorren la noción: a) entre un nivel teórico particular (histórico y específico) y universal (transhistórico y abstracto); b) entre un carácter descriptivo (analítico-teórico) y uno prescriptivo (estratégico-político). Las conclusiones a las que hemos arribado confieren una importante ligazón entre la noción y el marco evolucionista del cambio social; siendo este último, en gran parte, la causa primera de sus tensiones internas y sus conflictos normativos.

Por ello, como parte de ese camino abierto, proponemos aquí la tarea de recuperar las lecturas críticas del esquema evolucionista del cambio social y la noción parsoniana de comunidad societal que han realizado dos importantes teóricos y comentaristas de su obra: Nicos Mouzelis y Jeffrey Alexander. Intentaremos rastrear, identificar y analizar los diversos núcleos problemáticos que observan y, en segunda medida, evaluar hasta qué punto sus propuestas permiten superar los conflictos del marco evolucionista y/o despejar a la comunidad societal de sus tensiones internas. 


\section{Mouzelis}

En el artículo titulado Modernity: a non-European conceptualization (1999), Mouzelis realiza una profunda revisión del trabajo evolucionista parsoniano, que había abordado anteriormente en su obra central: Sociological Theory: What Went Wrong? (1995). Su intención es revisar la tendencia eurocéntrica de los conceptos de modernidad y modernización, tanto en los escritos clásicos de Parsons como en las revisiones críticas de Giddens o Stuart Hall; la "tendencia de ver las trayectorias (pasado, presente y futuro) de desarrollo no-occidentales como imitaciones de un patrón específicamente occidental de desarrollo" (Mouzelis, 1999: 141).

Mouzelis busca reconsiderar el problema del uso del concepto de modernidad y, por ello, aunque acepta las críticas de la teoría social anti-eurocéntrica, desecha sus propuestas constructivas. Están en lo cierto al considerar que el devenir de las sociedades no debe conceptualizarse aceptando la universalidad (o incluso la benevolencia) de patrones institucionales específicamente occidentales, pero el extremo relativismo (moral y cognitivo) que presentan las propuestas de estas escuelas teóricas ponen en peligro al concepto mismo de modernidad (Mouzelis, 1999: 142).

La solución al problema requiere, por lo tanto, de una posición intermedia, que reconozca los factores universales de la modernidad sin limitarlos a patrones de desarrollo derivados de modelos-ideales occidentales. Una concepción que permita describir las trayectorias específicas de países periféricos o nocapitalistas sin imposibilitar el análisis de las características distintivas y universales de la modernidad.

Es especialmente llamativo que el modelo eurocéntrico que Mouzelis intenta reformular ha sido identificado como uno de los problemas centrales de la teoría parsoniana por numerosos autores (Gouldner, 1973; Granovetter, 1979; Fanelli, 1986; Giddens, 1995). En particular, la idealización del estándar cultural norteamericano en tanto ideal evolutivo, base de complejos 
presupuestos evolucionistas que Parsons acepta sin reformular (Girola, 2010). Esta concepción, de la que derivan las tensiones internas de la comunidad societal, ha llevado a Parsons a suponer un proceso que uniría linealmente, en forma de etapas, la diferenciación, la integración, la inclusión y la justicia social ${ }^{3}$.

Mouzelis intenta, sugerentemente, superar este problema mediante la recuperación y reelaboración del propio trabajo parsoniano. Fundamentalmente a través de la concepción de universales evolutivos y el proceso de diferenciación estructural. Se basa, por ende, en una perspectiva analítica centrada en la Integración Sistémica (Lockwood, 1964), no sin antes señalar que el sobre-énfasis que Parsons ha colocado en esta perspectiva (en detrimento de la Integración Social) es el mayor problema de su teoría: "la agencia (particularmente, la agencia colectiva) desaparece o es representada de forma pasiva, como el producto de procesos sistémicos de diferenciación funcional-estructural" (Mouzelis, 1999: $144)^{4}$. En conclusión, Mouzelis acepta la diferenciación teórica que propone Lockwood y se limita al análisis de los problemas de la perspectiva de Integración Sistémica de Parsons.

La crítica central es que la linealidad evolutiva que propone Parsons en sus trabajos (diferenciación > inclusión > generalización de valores $>$ aumento de la capacidad adaptativa del sistema $)^{5}$ no se da necesariamente de esa forma, o incluso po-

3 El mismo problema convoca a Alexander (2005), que realiza una crítica teórico-política de la comunidad societal, exponiendo la confusión de Parsons entre "integración" y "justicia". Abordaremos su lectura en la segunda sección de este trabajo.

4 Mouzelis comparte esta crítica con numerosos comentaristas del trabajo parsoniano, desde los tempranos trabajos de los teóricos del conflicto (Dahrendorf, 1958; Rex, 1977) y los propios herederos de Parsons (Merton, 1968) hasta los neofuncionalistas (Alexander 1990a, 1990b; Colomy, 1990). Superar la dominación de la perspectiva sistémica (recuperación de la teoría de la acción social) es también parte central del trabajo de Schutz (1974), Garfinkel (2006), Giddens (1995, 1997) y Bourdieu (2007), entre otros.

$5 \mathrm{Al}$ mantenernos dentro de una perspectiva de Integración Sistémica, cada uno de estos conceptos (por ejemplo el de inclusión) refiere siempre a partes [parts] o 
dríamos decir, sólo se ha dado así de forma aislada y rudimentaria ${ }^{6}$ " La diferenciación, incluso fuertemente institucionalizada, no siempre se relaciona con los otros tres aspectos del desarrollo evolutivo en la forma en que Parsons lo insinúa" (Mouzelis, 1999:145). Aunque, sin lugar a dudas, esta enorme excepción en los procesos de modernización no puede ser tomada como el ideal (o progreso "normal") del desarrollo evolutivo.

En primer lugar, Mouzelis propone que ciertas partes diferenciadas del sistema pueden tomar formas balanceadas [balanced] o desbalanceadas [unbalanced] de inclusión. Es decir, que pueden ser integradas de forma tal que la lógica de una de las unidades diferenciadas domine la de otra ${ }^{7}$. Claramente, no pueden ser definidos como procesos regresivos de la forma en que solía hacerlo Parsons. Lejos de suponer una lógica de in-diferenciación de las partes, es decir, el regreso de partes a segmentos unificados, significa que las unidades se mantienen separadas y esa separación es institucionalizada aunque no poseen lógicas autónomas de funcionamiento. En palabras de Mouzelis (1999: 146):

La diferenciación formal refiere a la separación institucional de las partes o unidades del sistema, mientras que la diferenciación substantiva refiere al problema de la autonomía de las partes diferenciadas.

unidades [units] del Sistema, es decir, a complejos institucionales [institutional complexes]. No debe confundirse con la "integración" o "inclusión" de grupos o actores. La confusión de ambos aspectos, mucha veces dentro del propio trabajo parsoniano, es parte central de la crítica que realiza Alexander (2005) a la noción de comunidad societal.

6 Mouzelis se refiere al período de segunda posguerra de las democracias occidentales y la "breve transición del capitalismo liberal al social-democrático", que retrata como "un tímido desarrollo de una modernización multilógica o balanceada" (1999: 147).

7 Mouzelis (1999: 146) ejemplifica este caso con la creciente “dominación de la lógica de mercado/management" por sobre la lógica autónoma del "sistema británico de educación universitaria" durante la era Thatcher. 
Únicamente la autonomía de las partes diferenciadas definiría un proceso multilógico o balanceado de integración. Por el contrario, Parsons parece tomar por sentado esta autonomía como consecuencia inevitable de los procesos de diferenciación y especialización de las partes.

De aquí que el proceso complementario de generalización de valores sólo pueda darse en el caso de una integración balanceada de las unidades diferenciadas. $\mathrm{Si}$, por el contrario, la lógica de una de las partes domina al resto, la diferenciación puede ser institucionalizada de forma "particularista". Mouzelis acierta al subrayar que, incluso en las trayectorias modernas occidentaleuropeas, los procesos de inclusión no han sido "tan balanceados como Parsons insinúa" (1999: 147). La dominación del capitalismo liberal ha supuesto la inclinación hacia una lógica económica de productividad que minó seriamente la autonomía de los valores de los demás sub-sistemas.

Por último, pero no menos importante, nos encontramos ante el problema del aumento de capacidad adaptativa [adaptive upgrading] en la teoría evolucionista de Parsons ${ }^{8}$. Creemos que Mouzelis desarrolla dos críticas al respecto: una más específica y explícita; otra más implícita y general. Comenzaremos por la primera. Si la diferenciación puede darse a través de una integración monológica o multilógica y, por ende, no se encuentra ligada necesariamente (sino más bien al contrario) con la generalización de valores, entonces "tampoco se encuentra ligada esta última con el aumento de capacidad adaptativa" (Mouzelis, 1999: 147). Esto es claro si se acepta los casos de desarrollo que muestra Mouzelis, particularmente el de Corea, Taiwán o Japón, sociedades que tipifican una "inclusión des-balanceada" con fuerte presencia de los valores del subsistema político "patriarcal-autoritario" pero un importante desarrollo económico. Por ello, es difícil aceptar

8 Una crítica específica y profunda de este problema puede hallarse en Granovetter (1979). 
la suposición parsoniana de que las formas liberal-democráticas de gobierno, típicamente occidentales, son condición necesaria para un aumento de la capacidad adaptativa en el mundo moderno9. En este sentido, la generalización de formas políticas democráticas ha tenido "menos que ver con la inevitabilidad de las condiciones estructurales de la modernidad tardía que con la explicación coyuntural de la derrota de las fuerzas del eje y el surgimiento de la hegemonía norteamericana luego de la segunda guerra mundial" (Mouzelis, 1999: 147-48). Aquí se desliza un fuerte comentario respecto a la falta de ambivalencia e historicidad en la forma en que Parsons define la modernidad, resultado de su enfoque general evolutivo ${ }^{10}$. Una necesaria historización mostraría, por ejemplo, que incluso mayor diferenciación difícilmente conlleve un aumento de la capacidad adaptativa en cualquien situación o coyuntura histórica ${ }^{11}$.

Esto nos lleva a la segunda crítica, de orden más general, que Mouzelis no explicita pero lleva adelante en la sección constructiva de su escrito. Cuando propone una compleja pero profunda forma de concebir los procesos de modernización, inmediata e implícitamente, los conceptos de Parsons son reapropiados y reconceptualizados. Dentro del programa de Mouzelis, la comparativa evolutiva de Parsons (1974 [1966], 1971), que contenía un importante horizonte político-normativo, deja de tener sentido.

\footnotetext{
9 Por el contrario, como bien señala Mouzelis (1999: 148), en la coyuntura global presente, las formas liberales-democráticas "combinan crisis económicas crónicas [economic bottlenecks] con creciente desigualdad y periferialización socio-económica de las clases menos aventajadas".

10 En la segunda sección, mostraremos cómo este problema de la "ahistorización del modelo evolutivo" y la "idealización del orden normativo" se encuentran ligados a su tendencia creciente de limitar los problemas societales a problemas de Integración Sistémica.

11 Mouzelis (1999: 148) lo deja en claro recuperando a Bendix y Moore; pero específicamente a través de Hobsbawm y su comparativa entre el desarrollo conflictivo de Inglaterra en el siglo XIX (con un ya avanzado proceso de diferenciación) y el salto cualitativo de Alemania al incorporar fácilmente nuevas tecnologías (capacidad de inclusión por poseer menor diferenciación estructural).
} 
Por ello, su caracterización universal de la modernidad depende de las nociones de universal evolutivo y capacidad adaptativa, pero ambas están vaciadas del contenido normativo y prescriptivo que poseían en la teoría parsoniana.

En el primer caso, la existencia de la modernidad puede definirse mediante dos factores: a) la movilización de recursos y la "incorporación al centro"12; b) un nivel sin precedentes de diferenciación estructural ${ }^{13}$. Ambas cuestiones no se deben a un proceso de acumulación evolutiva y progresiva ni son evaluadas normativamente como superiores o necesariamente prevalecientes a largo plazo. Por el contrario, Mouzelis les otorga un origen profundamente histórico y, por otra parte, normativamente ambivalente, a todas las características transculturales de la modernidad (sociales, culturales, económicas y políticas) ${ }^{14}$. Estos universales evolutivos no tienen una lógica comparativa o normativa, se refieren a las características compartidas (transculturales) de una etapa específica (y global) de la organización social. Por ello, se destaca que no refieren a una "particularidad única" de Occidente sino a factores sin los cuales "ninguna sociedad puede sobrevivir en el presente orden mundial" (Mouzelis, 1999: 151). Lo que es mucho más importante, al comprender la importancia de la primera crítica, es claro que estos factores no tienen por qué seguir un patrón unilateral u "occidentalista".

12 Con "incorporación al centro", Mouzelis se refiere al cambio de lealtades de la población, desde comunidades locales-tradicionales a la "comunidad imaginada" del Estado-Nación.

13 Cada uno de los factores corresponde a dos tipos de análisis, el primero a una perspectiva de Integración Social, el segundo a una perspectiva de Integración Sistémica.

14 Aunque no lo desarrollaremos aquí, se refieren al desarrollo de tecnologías culturales (la imprenta, entre otras) que permitieron la educación y la lectura masiva (L); la construcción de tecnologías nacionales de administración, burocracia, vigilancia y violencia $(G)$; la definición de mercados nacionales, la separación del productor directo y los medios de producción y las tecnologías fabriles de la Revolución Industrial (A); la definición de arenas nacionales centralizadas del cuidado, salud y administración de la población (I). 
Por otra parte, quizás de forma obvia, el concepto de capacidad adaptativa adquiere una relevancia puramente moderna. Mouzelis lo acepta pero lo redefine como "la generación de recursos que le permiten a una sociedad evitar los típicos embudos [bottlenecks] del desarrollo tardío". Pasa a ser un problema económico e interno a los problemas de los Estados-Nación modernos. Esto marca una enorme diferencia con los textos de Parsons, donde la capacidad adaptativa era el eje a través del cual comparar distintos tipos de sociedades históricas y definir el correcto devenir del desarrollo evolutivo de la humanidad. La redefinición de Mouzelis no es ingenua.

Toma en cuenta, profundamente, la crítica de Alexander (1990a: 2):

Describir meramente a la diferenciación de forma general, la hace parecer un mecanismo automático de equilibramiento, algo que ocurre en cualquier momento en que son necesarios ajustes ante el conflicto y las tensiones.

Por el contrario, Mouzelis reconsidera la importancia de los acontecimientos históricos en la definición de un orden social específico y en la generalización de ciertos factores trasnacionales ${ }^{15}$. Supera entonces el problema central de la teoría de Parsons, que no se halla en el concepto de diferenciación o en la incapacidad de aceptar la creciente diferenciación institucional del Estado-Nación, sino, por el contrario, en aceptarlo como un proceso acumulativo y auto-regulador que tiende a una mayor adaptación del sistema.

La crítica general de Mouzelis inhabilita una comparativa entre distintos tipos de sistemas sociales epocales al definir los

15 Por ejemplo, la importancia de la hegemonía internacional de Estados Unidos desde la segunda guerra mundial y la definición de patrones económicos neoliberales para el resto de los Estados. Además, de la relación entre ese proceso político internacional y la creciente dominación de la lógica productiva-mercantil dominando, no solo la economía (A), sino el resto de los subsistemas sociales (G, I, L). 
universales evolutivos a través de su propia unicidad histórica, y demuestra que el occidentalismo de la teoría parsoniana se debe, en gran medida, a una aceptación acrítica de la linealidad entre los componentes de su marco evolucionista. En definitiva, los "cuatros factores del desarrollo evolutivo" que individualiza Parsons varían independientemente (la diferenciación estructural es la base y no la cúspide de los procesos de modernización) y dependen, en primera instancia, de procesos histórico-políticos específicos, de relaciones conflictivas entre actores y grupos sociales que deben ser analizados (no presupuestos) desde una perspectiva teórica de Integración Social.

\section{Alexander}

Discípulo de la sociología parsoniana, tanto en su interés por renovar la propuesta teórica del cambio social que propone el funcionalismo como en el estudio de las dimensión culturalnormativa de la integración social (1990), Alexander ha realizado una exhaustiva revisión de la obra tardía de Parsons. Se interesó, especialmente, en la teoría general de la diferenciación y el tratamiento que allí recibe el problema de la solidaridad social. Esta relectura crítica significó el punto de partida para reformular las bases teóricas del paradigma funcionalista. Partiendo del marco explicativo general sistematizado por Parsons, el neo-funcionalismo se ocupó de realizar un fuerte apuntalamiento de la teoría de la diferenciación para establecer una explicación más comprensiva del cambio social ${ }^{16}$. Finalmente, este camino supuso un alejamiento completo del marco funcionalista y el inicio de una nueva línea teórica en sociología cultural. En esta última etapa

16 Un desarrollo que incluyó la reconfiguración de sus presupuestos teóricos, el tratamiento analítico de las particularidades históricas de cada sociedad y del impacto desigual producido por los grupos sociales, la concepción del poder, el conflicto y la contingencia, así como la revisión de su carga ideológica (Colomy, 1990: 468-469). 
se ubica el trabajo que Alexander dedicó, de forma exclusiva, a revisar el concepto de comunidad societal a la luz del conjunto de la obra parsoniana. La profundidad de la crítica que allí formula, de todas formas, sólo puede ser comprendida adecuadamente revisando algunas de las observaciones críticas que el neo-funcionalismo señala en la teoría general de la diferenciación.

A contramano de un importante número de trabajos (Treviño, 2001; Gerdhardt, 2001, 2002; Sciortino, 2004, 2005, 2010) que en los últimos años han recuperado la importancia y el peso potencial de la noción para la teoría social contemporánea, Alexander presenta un análisis sumamente crítico en su artículo Contradictions in The Societal Community: The Promise and Dissapointment of Parsons Concept (2005). El eje del planteo radica en que la noción presenta una tensión inherente y no explícita entre "inclusión" e "integración" que, en términos teóricos, impone fuertes restricciones a la capacidad heurística del concepto y, en términos normativos, supone una concepción antidemocrática de la organización social. Alexander cree que esta contradicción tan problemática del concepto tiene un arraigo profundo en la propia postura ambivalente de Parsons respecto al orden y la normatividad, tanto ideológica como presuposicional. Precisamente, lo más sugerente de este planteo y de la argumentación subsiguiente es definir las implicancias ideológicas de Parsons a través de su propio marco analítico. En este sentido, Alexander deja en claro que la principal causa de las tensiones conceptuales internas y los conflictos normativos de la comunidad societal remiten ineludiblemente a problemas enquistados en la teoría evolutiva que le sirve de marco.

Parsons tenía especial interés en desarrollar un teoría más sofisticada del subsistema integrativo (I) del modelo AGIL, creado a finales de los años ‘ 50 . Retomando la insistencia teórica durkhemiana de considerar la solidaridad como una dimensión distintiva de la vida social, Parsons sistematiza esa esfera funcional de la sociedad en la comunidad societal, concebida de forma autónoma 
pero en igualdad de condiciones que la economía (A), las orientaciones de valor (L) y la política (G). Asimismo, Parsons deseaba incorporar la posibilidad teórica de pensar la dimensión democrática de las sociedades modernas. Para ello, define el tipo de solidaridad prototípico de la sociedad moderna (que la distingue de la solidaridad primordial de la sociedad tradicional) a partir de la concepción de ciudadanía social-democrática propuesta por T. H. Marshall.

En este sentido, la elaboración del concepto supone el desafío de dar una respuesta conjunta a los reclamos de integración social y las posibilidades de justicia social que atraviesan las sociedades modernas. A través de la comunidad societal se presenta, entonces, el marco analítico buscado para postular una solidaridad social que asegure la integración sin perjudicar (por el contrario, favoreciendo) la creciente diferenciación institucional y pluralidad cultural características de las sociedades modernas. Para sostener ese "plus" (analítico) de complejidad y (normativo) de igualdad que significa ingresar en la modernidad, Parsons caracteriza a la comunidad societal como un conjunto de referencias culturales y normas morales lo suficientemente fuertes y motivacionalmente vinculantes como para garantizar mínima solidaridad y, al mismo tiempo, lo suficientemente abstractos y universales como para ser apropiados-interiorizados por distintos grupos (Alexander, 2005: 96).

De esta manera, Parsons unifica, bajo un mismo concepto, las dimensiones de integración y justicia como si estos dos problemas sociales no remitieran a distintos procesos (Alexander, 2005: 96-97). Como plantea Alexander, la existencia generalizada de principios morales internalizados por todos los grupos y de normas sociales institucionalizadas, que otorga cohesión y orden a una sociedad, no implica como correlato necesario que la estructura social sea igualitaria y democrática:

El orden normativo per se no es democrático. Es meramente cultural. [...] Puede lograr integración, indu- 
ciendo cooperación, consenso y aceptación generados internamente. Sin embargo, dicha integración sólo será hegemonía (Alexander, 2005: 97).

Esta falta de atención en la distinción entre integración y justicia social se manifiesta, por igual, tanto en el tratamiento histórico-político como en la definición teórica que Parsons realiza de la comunidad societal, porque la causa del problema se encuentra más abajo, en los fundamentos presuposicionales de su marco evolutivo de la diferenciación estructural.

Parsons define los principios fundamentales de su esquema de forma excesivamente abstracta, esquemática y unidireccional, dejando fuera de su base teorética los rasgos ambivalentes, contradictorios y contingentes del mundo social. La crítica a Parsons que recuperan Alexander (1990a) y Colomy (1990: 466467) puede ser sintetizada de la siguiente manera: el cambio social es descripto de forma general por una "tendencia matriz" a la diferenciación en unidades más especializadas; se precipita por un mecanismo automático de equilibramiento, algo que ocurre en cualquier momento en que la ineficiencia funcional provoca una tensión estructural y, como resultado, la mayor complejización implica siempre un incremento en la capacidad adaptativa del sistema. El cambio es entendido unívocamente como un proceso acumulativo y autorregulado, es decir, antecedido por una situación de estabilidad social a la cual siempre se regresa ${ }^{17}$. Como resultado, un elevado nivel de diferenciación se corresponde (mecánicamente, es justo enfatizar) con la generalización de valores, mayor inclusión social y la emergencia de instituciones integrativas especializadas (Parsons, 1974 [1966], 1971).

El excesivo nivel de abstracción que Parsons otorga a la diferenciación mostró los signos de su agotamiento cuando la misma

17 Lo que Fanelli (1986) denomina cambio "dentro del sistema". 
teoría general pretendía poder explicar el cambio en toda institución y en toda esfera social (Alexander, 1990a: 8).

Su carencia de análisis específicos de las fases históricas, su falla en tratar los niveles estructural e institucional, su negación de los procesos; todo ello contribuyó al aura ahistórica e idealista de esta muy sofisticada pero prototípica teoría de la modernización (1990a: 10-11).

Alexander señala que esta concepción idealista de la esfera normativa de la sociedad acarrea graves consecuencias para el análisis de la integración. Por un lado, el pasaje de un vínculo solidario comunitario a uno civil se da por descontado, como un avance progresivo y necesario de la modernización, como mero reflejo de otros procesos de diferenciación.

Este estándar [...] ha sido usualmente aplicado de manera artificial y lineal incluso por aquellos teóricos que tomaron en serio el problema de la integración. De Hegel y Tocqueville a Parsons, la transición desde una solidaridad primordial a una civil ha sido imaginada como rígidamente entrelazada con la transformación económica y política (Alexander, 1990b: 269-270).

Por el contrario, la estructura de solidaridad tiene mecanismos particulares y $\operatorname{concretos}^{18}$. Además, cuando se pierde de vista la realidad histórica, no se tiene en cuenta que la diferenciación no es un proceso homogéneo. Por consiguiente, no se percibe que la solidaridad se desarrolla en diferentes esferas sociales a distinto tiempo, y estos avances y/o demoras tienen repercusiones enormemente complejas en el desarrollo societal (1990b: 271).

18 Alexander menciona, entre otros procesos, el incremento de la movilidad geográfica y cultural, la urbanización, la educación secular y los rituales civiles cada vez más consensuados. 
La segunda consecuencia analítica que produce la idealización del proceso de integración refiere a pasar por alto un aspecto fundamental de cualquier estructura normativa nacional: la existencia de un núcleo [core] histórico. Aunque este grupo social fundador establezca un marco de referencia político-nacional altamente diferenciado, establecerá necesariamente, al mismo tiempo, la preeminencia de ciertas cualidades culturales primordiales. Por lo tanto, aquellos miembros que no formen parte del grupo nuclear, aunque puedan acceder plenamente a los derechos civiles y acumulen elevados niveles de participación institucional, quedan expuestos a la posibilidad de no poseer membresía plena en la comunidad nacional o a verla súbitamente restringida. Según Alexander (1990b: 271-272): “esta tensión entre solidaridad original y civil debería informar cualquier teoría de la inclusión de sociedades industriales".

En el caso del estudio Full Citizenship for the Negro American? (Parsons, 1965), el problema del núcleo [core] es especialmente interesante. Aquí, a diferencia de otros trabajos ulteriores, Parsons se preocupa específicamente por la integración en tanto "inclusión" de grupos sociales, en base a una mayor "igualdad de oportunidades". En ese sentido, cuando se refiere al "núcleo" de la comunidad norteamericana, habla de un estrato poblacional históricamente diferenciado (WASP) que sostiene los valores centrales y lleva adelante las decisiones gubernamentales en Estados Unidos. El proceso de integración refiere, en parte, a las luchas por los derechos civiles que posibilitan la ampliación de ese "núcleo". En este estricto sentido, el acercamiento de Parsons al problema de la comunidad societal supondría un tratamiento empíricamente orientado e históricamente más preciso de los movimientos en la estructura normativa nacional moderna, como lo plantea Alexander. No sería desacertado, entonces, considerar que Parsons sutilmente trasciende la abstracción y rigidez de su propio esquema analítico, al considerar el conflicto entre grupos sociales en la definición del orden normativo (plano de Integra- 
ción Social $)^{19}$. Sin embargo, esto no implica resolución alguna al problema de indistinción entre integración e justicia en la definición de comunidad societal.

En cuanto nos alejamos hacia una perspectiva más general del desarrollo teórico de la noción, el rastro idealista constitutivo de la perspectiva evolucionista vuelve a presentarse. En este mismo escrito, encontramos una primera definición que ya presenta una tensión entre carácter descriptivo y prescriptivo: en las sociedades "avanzadas", existe (o debe existir) un aspecto nuclear que permite (o debe permitir) regular procesos integrativos del todo social frente a la heterogeneidad en la composición cultural de sus grupos sociales ${ }^{20}$. La necesidad teórico-evolutiva de resolver el problema de integración se superpone con la urgencia política por allanar el camino hacia la incorporación completa de los "americanos negros" en la ciudadanía nacional americana. Así, la comunidad societal adquiere un "sentido utópico-normativo" de primer orden (de Marinis, 2011) y, como bien plantea Alexander (2005: 96), Parsons tiende a idealizar la conexión entre integración y justicia.

Para justificar su afirmación de que en la propia definición abstracta Parsons sienta las bases no-democráticas de la comunidad societal, Alexander realiza un análisis bastante problemático de las definiciones formuladas en los libros evolucionistas de Parsons. El objetivo de sus señalamientos es mostrar cuál es la

19 Es un argumento compartido por varios autores (Chernillo, 2004, 2010; Turner, 1999; Alexander, 1990) que los estudios "empíricamente orientados" de Parsons permiten acceder a una complejidad de análisis que no hallamos en sus textos teóricos sistemáticos, particularmente en lo que refiere a la concepción de la modernidad y sus características ambivalentes.

20 "La comunidad societal es el foco de lealtades que no necesita ser absoluto, además no lo puede ser, pero que requiere alta prioridad entre las lealtades de los miembros. Para ocupar esta posición, la estructura de asociación debe estar en acuerdo con los valores comunes de la sociedad: los miembros están comprometidos a ella porque ésta implementa sus valores y organiza sus intereses en relación a otros intereses" (1965: 424. Nuestras cursivas). 
base presuposicional que hace de la propia definición abstracta una herramienta teórica sesgada, basada en una concepción de la integración que se funda en la hegemonía cultural. Alexander (2005: 98) se esfuerza por enfatizar que la definición de Societies (1974 [1966]: 10), si bien refiere a "un orden normativo pautado a través del cual la vida de la población se organiza colectivamente", también incluye una equiparación del núcleo con un "grupo per se" que "define la naturaleza de la membresía y adjudica el reforzamiento de los significados normativos". Y para destacar ese aspecto excluyente de la definición, Alexander concluye que con "la vida de la población" Parsons se refiere a "individuos y grupos que son administrativa o funcionalmente parte del Sistema Social pero que están fuera del núcleo".

En verdad, Parsons no define al "núcleo" de esa forma en Societies. Por el contrario, lo define específicamente como el "orden normativo" de la sociedad. Alexander se equivoca entonces al equiparar diversas definiciones que Parsons utiliza. Sin embargo, ese error no inhabilita el centro de su crítica a la comunidad societal como un concepto que supone una concepción restringida del orden normativo y puede movilizar argumentos conservadores. Tan sólo creemos que la crítica no debe dirigirse a la presencia o no de un elemento específico de la definición sino al espacio en que esa definición se inscribe, es decir, en los mecanismos conceptuales del marco evolucionista general. Desde una mirada panorámica del conjunto de la obra tardía de Parsons, se puede observar un corrimiento del concepto de núcleo [core] -análogo al proceso de abstracción de la teoría evolucionista parsoniana-. En Full Citizenship for the Negro American? (1965), Parsons utiliza la noción de "núcleo" como "colectivo social predominante o hegemónico". Sin embargo, a partir de Societies (1966), la noción de "núcleo" vira su significado hacia "estructura normativa o legal de la sociedad".

Por ende, la lectura de sus textos más abstractos sobre el papel de la comunidad societal desnuda la idealidad del "orden nor- 
mativo" que señala Alexander. Parsons pierde progresivamente su interés en los procesos de Integración Social y se limita a procesos de Integración Sistémica, confundiendo "integración normativa" con "justicia social" u "orden social equitativo". A veces, sencillamente diluye los primeros en los segundos. Es innegable que, si aceptamos la propia lectura socio-histórica de Parsons, la definición de pautas y normas de la "estructura normativa o legal" de la sociedad se encuentra asociada a un proceso inequitativo y desigual, basado en la existencia separada de un "nucleo societal" con poder diferenciado.

\section{Conclusión}

Hemos intentado realizar una aproximación simultánea a dos lecturas críticas del marco teórico del Parsons cibernético-evolucionista. Dos revisiones de la última porción de la obra parsoniana que, aunque parten de objetivos e intereses diversos, poseen fuertes puntos en común. Fundamentalmente, comparten la identificación de problemáticas en el marco teórico evolucionista del cambio social que nos interesaba trabajar aquí. Ambos autores remarcan los problemas del uso que Parsons otorga al concepto de diferenciación; la idealidad del proceso modernizador (en tanto define una continuidad lineal desde la diferenciación hasta el desarrollo, la inclusión y la justicia social); la subordinación de los problemas de la agencia, los conflictos entre sujetos y grupos, y la definición desigual del orden normativo.

Estos problemas generales provocan una falta de ambivalencia en su concepción teórica de la modernidad y, por ende, del rol que ha de cumplir la comunidad societal como foco de integración de la sociedad. Creemos que Alexander define con claridad las complicadas tendencias conservadoras de la noción, en tanto arrastra una confusión inherente (y no resuelta) del evolucionismo parsoniano: una tensión entre elementos descriptivos y normativos y, centralmente, entre elementos particularistas y abstractos del análisis social. En esta última etapa, Parsons resigna 
crecientemente (desde sus escritos "empíricamente orientados" de posguerra hasta los textos sistémicos más abstractos de sus últimas dos obras) los problemas de la Integración Social y, lo que es peor, tiende a transformarlos (o subsumirlos sin más) en problemas de Integración Sistémica. Por esa razón (su concepción lineal e ideal de los procesos de cambio social), Parsons resignó el problema de la "justicia social" ante el de la "integración": una confianza ciega en el devenir unívoco de la modernidad lo imposibilitaba a descubrir sus rasgos más oscuros, su persistente ambivalencia, sus crudos procesos de estratificación social, y la batalla continua por la hegemonía cultural.

\section{Bibliografía}

Alexander, Jeffrey (1990a): “Differentiation Theory: Problems and Prospects". En: Differentiation Theory and Social Change, Alexander, Jeffrey and Paul Colomy (eds.). New York, Columbia University Press.

Alexander, Jeffrey (1990b): "Core Solidarity, Ethnic Out Groups, and Social Differentiation". En: Differentiation Theory and Social Change, Alexander, Jeffrey and Paul Colomy (eds.). New York, Columbia University Press.

Alexander, Jeffrey (2005): “Contradictions in the Societal Community: The Promise and Disappointment of Parsons Concept". En: After Parsons: A Theory of Social Action for the Twenty First Century, Fox, Renée C.; Victor Lidz y Harold J. (eds.). New York, Russell Sage Foundation.

Bourdieu, Pierre (2007): El sentido práctico. Buenos Aires, Siglo XXI.

Chernilo, Daniel (2004): "El rol de la 'sociedad' como ideal regulativo". En: Cinta de Moebio, Revista Electrónica de Epistemología de Ciencias Sociales, No 21.

Chernilo, Daniel (2010): Nacionalismo y cosmopolitismo. Chile, Universidad Diego Portales.

Colomy, Paul (1990): "Revisions and Progress in Differentiation Theory". En: Differentiation Theory and Social Change, Alexander, Jeffrey and Paul Colomy (eds.). New York, Columbia University Press. 
Dahrendorf, Ralf (1958): "Towards a Theory of Social Conflict". En: Journal of Conflict Resolution, Vol. 2 (170-183).

de Marinis, Pablo (2010): “La comunidad societal en Talcott Parsons: un ejercicio para poner a prueba ciertas hipótesis de lectura acerca de la historia del concepto de comunidad en la teoría sociológica". En: VI Jornadas de Sociología de la Universidad Nacional de La Plata (actas en formato CD). Buenos Aires, UNLP.

de Marinis, Pablo (2011): “La teoría sociológica y la comunidad: clásicos y contemporáneos tras las huellas de la 'buena sociedad'". En: Entramados y perspectivas, Revista de la Carrera de Sociología, Facultad de Ciencias Sociales, UBA, Año I, Vol. 1, $\mathrm{N}^{\mathrm{o}}$ 1. Buenos Aires, FSOC.

de Marinis, Pablo (comp.) (2012): Comunidad: estudios de teoría sociológica. Buenos Aires, Editorial Prometeo.

Fanelli, Ana M. García (1986): Talcott Parsons y la Teoría del Cambio Social. Buenos Aires, Ides.

Garfinkel, Harold (2006): Estudios en etnometodología. España, Anthropos.

Gerhardt, Uta (2001): "Parsons analysis of the societal community". En: Talcott Parsons Today. His Theory and Legacy in Contemporary Sociology, Treviño, A. Javier (ed.). Lanham, Rowman \& Littlefield Publishers, Inc.

Gerhardt, Uta (2002): Talcott Parsons. An Intellectual Biography. Cambridge, Cambridge University Press.

Giddens, Anthony (1995): La constitución de la sociedad. Buenos Aires, Amorrortu.

Giddens, Anthony (1997): Las nuevas reglas del método sociológico: Crítica positiva de las sociologías comprensivas. Buenos Aires, Amorrortu.

Girola, Lidia (2010): “Talcott Parsons: a propósito de la evolución social”. En: Sociológica, Año 25, No 72 (169-183).

Gouldner, Alvin (1973): La crisis de la sociología occidental. Buenos Aires, Amorrortu.

Granovetter, Mark (1979): “The Idea of 'Advancement' in Theories of Social Evolution and Development". En: The American Journal of Sociology, Vol. 85, N³ (489-515). 
Mouzelis y Alexander: lecturas críticas del esquema evolucionista de Talcott Parsons - Natalio Pagués y Nicolás Rubí

Lockwood, David (1964): "Social and System Integration". En: Explorations in Social Change, Zollschan G. K. y W. Hirsch (eds.). London, Routledge.

Merton, Robert K. (1968): "Estructura social y anomia". En: Teoría y estructura sociales, Cap. VI (209-239). México, Fondo de Cultura Económica.

Mouzelis, Nicos (1997): "Social and System Integration: Lockwood, Habermas, Giddens". En: Sociology, Vol. 31 No 1 (111-119).

Mouzelis, Nicos (1999): "Modernity: a non-European conceptualization", en British Journal of Sociology, Vol. 50 N 1 (141-159).

Pagués, Natalio y Rubí, Nicolás (2010): “Comunidad Societal y Cambio Social: tensiones evolucionistas en el Parsons tardío". En: VI Jornadas de Sociología de la Universidad Nacional de La Plata (actas en formato CD), Buenos Aires.

Pagués, Natalio y Rubí, Nicolás (2012): “Comunidad Societal y Cambio Social: tensiones evolucionistas en la obra de Parsons". En: Comunidad: estudios de teoría sociológica, de Marinis, Pablo (comp.). Buenos Aires, Editorial Prometeo.

Parsons, Talcott (1964): "Evolutionary Universals in Society". En: American Sociological Review, No3 (339-358).

Parsons, Talcott (1965): "Full Citizenship for the Negro American? A Sociological Problem". En: Daedalus, Vol. 94, No. 4, The Negro American, MIT Press (1009-1054).

Parsons, Talcott (1969 [1959]): “The Principals Structures of Community". En Politics and Social Structure. New York, The Free Press.

Parsons, Talcott (1969): Politics and Social Structure. New York, The Free Press.

Parsons, Talcott (1971): The System of Modern Societies. New Jersey, Englewood Cliffs.

Parsons, Talcott (1974 [1966]): La Sociedad. Perspectivas evolutivas y comparativas. México, Trillas.

Parsons, Talcott (1976 [1968]): "Sistemas Sociales", voz de la Enciclopedia Internacional de las Ciencias Sociales, Tomo IX. Madrid, Ediciones Aguilar (710-721).

Parsons, Talcott (1988 [1951]): El Sistema Social. Madrid, Alianza.

Parsons, Talcott (2007): American Society. A Theory of the Societal Commu- 
nity (Giuseppe Sciortino, ed.). Boulder, Paradigm Publishers.

Rex, John (1977): Problemas fundamentales de teoría sociológica. Buenos Aires, Amorrortu.

Schutz, Alfred (1974): Estudios sobre teoría social. Buenos Aires, Amorrortu.

Sciortino, Giuseppe (2004): The Societal Community. Conceptual Foundations of a Key Action Theory Concept. Paper for the Parsons Conference in Kobe University.

Sciortino, Giuseppe (2005): “How Different Can We Be? Parsons's Societal Community, Pluralism, and the Multicultural Debate". En: After Parsons: A Theory of Social Action for the Twenty First Century, Renée C. Fox, Victor Lidz, y Harold J. Bershady (eds.). New York, Russell Sage Foundation (111-136).

Sciortino, Giuseppe (2010): “A single societal community with full citizenship for all: Talcott Parsons, citizenship and modern society". En: Journal of Classical Sociology, Vol. 10 (239-258).

Treviño, A. Javier (ed.) (2001): “Introduction: The Theory and Legacy of Talcott Parsons". En: Talcott Parsons Today. His Theory and Legacy in Contemporary Sociology. Lanham, Rowman \& Littlefield Publishers, Inc. 